\title{
Design and implementation of the next generation electron beam resists for the production of EUVL photomasks
}

Scott M. Lewis, Guy A. DeRose, Hayden R. Alty, Matthew S. Hunt, Jarvis Li, et al.

Scott M. Lewis, Guy A. DeRose, Hayden R. Alty, Matthew S. Hunt, Jarvis Li, Alex Werthiem, Trevor Fowler, Sang Kook Lee, Christopher A. Muryn, Grigore A. Timco, Axel Scherer, Stephen G. Yeates, Richard E. P. Winpenny, "Design and implementation of the next generation electron beam resists for the production of EUVL photomasks," Proc. SPIE 10810, Photomask Technology 2018, 108100N (3 October 2018); doi: 10.1117/12.2501808

Event: SPIE Photomask Technology + Extreme Ultraviolet Lithography, 2018, Monterey, California, United States 


\title{
Design and implementation of the next generation electron beam resists for the production of EUVL photomasks
}

\author{
Scott M. Lewis, ${ }^{\mathrm{a}, \mathrm{b} *}$ Guy A. DeRose, ${ }^{\mathrm{b}}$ Hayden R. Alty, ${ }^{\mathrm{a}}$ Matthew S. Hunt, ${ }^{\mathrm{b}}$ Jarvis Li, ${ }^{\mathrm{b}}$ Alex \\ Werthiem, ${ }^{\mathrm{b}}$ Trevor Fowler, ${ }^{\mathrm{b}}$ Sang Kook Lee ${ }^{\mathrm{b}}$, Christopher A. Muryn, ${ }^{\mathrm{a}}$ Grigore A. Timco, ${ }^{\mathrm{a}}$ Axel \\ Scherer, ${ }^{b}$ Stephen G. Yeates ${ }^{\text {a }} \&$ Richard E. P. Winpenny ${ }^{a} *$ \\ ${ }^{a}$ School of Chemistry and Photon Science Institute, The University of Manchester, Oxford Road, \\ Manchester M13 9PL, United Kingdom; E-mail: scott.lewis@,manchester.ac.uk or \\ Slewis2@Caltech.edu \\ b The Kavli Nanoscience Institute, California Institute of Technology, 1200 East California \\ Boulevard, 107 - 81, Pasadena, CA USA 91125.
}

\begin{abstract}
A new class of resist materials has been developed that is based on a family of heterometallic rings. The work is founded on a Monte Carlo simulation that utilizes a secondary and Auger electron generation model to design resist materials for high resolution electron beam lithography. The resist reduces the scattering of incident electrons to obtain line structures that have a width of $15 \mathrm{~nm}$ on a $40 \mathrm{~nm}$ pitch. This comes at the expense of lowering the sensitivity of the resist, which results in the need for large exposure doses. Low sensitivity can be dramatically improved by incorporating appropriate functional alkene groups around the metal-organic core, for example by replacing the pivalate component with a methacrylate molecule. This increases the resist sensitivity by a factor of 22.6 and demonstrates strong agreement between the Monte Carlo simulation and the experimental results. After the exposure and development processes, what remains of the resist material is a metal-oxide that is extremely resistant to silicon dry etch conditions; the etch selectivity has been measured to be $61: 1$.
\end{abstract}

Keywords: Metal Organic electron beam resist, electron beam lithography, 3D Secondary and Auger electron Monte Carlo Simulation

\section{INTRODUCTION}

The ability to write structures at the nanoscale using lithography underpins all modern, computer-based technology. The electronic devices we take for granted contain integrated circuits, the key component of which are fieldeffect transistors (FETs). These have reduced in size by a factor of two every two years over the past forty years, following "Moore's Law". The roadmap for the electronics industry now assumes that this constant reduction of size will continue, at least until the mid-2020s. Immersion $193 \mathrm{~nm}$ optical lithography (i193nm) is the current technique used to manufacture FinFETs, field-effect transistors with a shape resembling a fin. Unfortunately, this technique is reaching its physical limits in resolution. To counteract this, extreme ultra violet lithography (EUVL) has been promised to replace this technology. After two decades of outstanding research, numerous technical achievements, and multi-billion dollar investment, EUVL technology has only recently arrived into the research element of the semiconductor industry. While EUVL tools are being delivered, there is still a great need to develop new resist materials that will result in the creation of suitable EUVL photomasks. To achieve the $7 \mathrm{~nm}$ node that is expected to be in production by 2024 , the features on the photomask, which are presently produced using electron beam lithography (EBL), are required to be $30 \mathrm{~nm}$. Current e-beam resists cannot hit that specification, which means that new resists are needed in order to extract the full potential from EUVL.

Negative tone resists that are currently being used to fabricate photomasks for i193nm lithography are NEB22 (Sumitomo) and EVN30 (Shipley); they have a resolution of 40 and $50 \mathrm{~nm}$, respectively [1, 2]. This resolution is required because the $193 \mathrm{~nm}$ tools project the image onto photoresist and have lens reduction of $4 \mathrm{x}$. Thus, to achieve the $14 \mathrm{~nm}$ node, features on the photomask need to be $56 \mathrm{~nm}$. This is well outside the specification of $30 \mathrm{~nm}$ that is required for the production of EUVL photomasks. Another well-known issue presented by reducing feature sizes is that the

Photomask Technology 2018, edited by Emily E. Gallagher, Jed H. Rankin, Proc. of SPIE Vol.

10810, 108100N · C 2018 SPIE · CCC code: $0277-786 \mathrm{X} / 18 / \$ 18 \cdot$ doi: $10.1117 / 12.2501808$ 
required exposure dose to make them increases. This leads to longer write times, thus decreasing the throughput of photomask creation. To alleviate this issue, new EBL tools that utilise multiple beams are being designed to fabricate these photomasks. These tools have as many as 262,144 beams and have demonstrated a half-pitch resolution capability of $11 \mathrm{~nm}$ [3], but exposure times required for to exposure a full photomask are still too long. Therefore, new resist materials are required with higher sensitivities and higher resolution, and are the subject of this work.

Once the high-resolution pattern has been achieved, it must be transferred into the underlying substrate. This is usually achieved using an inductively-coupled plasma (ICP) etching technique. Etching nanostructures at dimensions that are less than $50 \mathrm{~nm}$ pitch, however, is very difficult because the probability of landing the neutral ions between features is extremely low [4]. This leads to a decrease in etch efficiency, which inherently decreases the etch rate and selectivity. To increase the etching efficiency, the ICP forward power can be increased, but this increases the etch rate of the resist, too, which would then require thicker resist to achieve the proper etch depth, which itself would require higher dose and result in reduced resolution. To avoid these interrelated issues, the ideal resist must be able to withstand the aggressive nature of the etching plasma. One method of achieving this is to incorporate metal materials into the resist. It has been shown that resists that are comprised of metal species exhibit small etch rates (because they have very little or no chemical reactivity with the gases that are used to etch the substrate) and consequently demonstrate large selectivities [4].

To address all of the above issues, we have developed a new class of resist materials that is based on a family of heterometallic rings [5]. Our resist material uses rigidity within the metal-organic molecule to achieve a very low density $(\rho=1.212)$ while exhibiting a large molecular weight $(2192 \mathrm{~g} / \mathrm{mol})$, which produces high resolution nanostructures. Figure 1a shows the resist molecule, established by the binding of eight chromium $(\mathrm{Cr})$ atoms (in green) to form a ringlike frame structure; its chemical formula is $\mathrm{Cr}_{8} \mathrm{~F}_{8}\left(\mathrm{C}_{5} \mathrm{H}_{9} \mathrm{O}_{2}\right)_{16}$, denoted henceforth as $\mathrm{Cr}_{8} \mathrm{~F}_{8}(\text { Pivalate })_{16}$. The fabrication of this structure is described elsewhere [6]. The exterior of the compound, composed entirely of tert-butyl groups (pivalates), gives the compound high solubility in non-polar solvents and allows it to be spun onto substrates, e.g., silicon and chromium. Upon exposure to electrons, a bond scission interaction occurs, resulting in the carbon and oxygen atoms in the pivalate molecule reacting to form $\mathrm{CO}_{2}$ gas, which volatilizes in the vacuum. This leaves behind a chromium oxide material that is insoluble in the developer solvent [4]. These resists write with very high resolution, but at the expense of lower sensitivity. To improve the resist sensitivity, we hypothesized that replacing the pivalate molecule with a methacrylate molecule (see Figure $1 \mathrm{~b}$ ) would lead to a reduction in the exposure dose without negatively impacting the resolution. The chemical formula of the resultant resist is $\mathrm{Cr}_{8} \mathrm{~F}_{8}\left(\mathrm{C}_{4} \mathrm{H}_{5} \mathrm{O}_{2}\right)_{16}$, denoted henceforth as $\mathrm{Cr}_{8} \mathrm{~F}_{8}\left(\mathrm{Methacrylate}_{16}\right.$. This resist has dangling alkene groups outside of the molecule that are free to activate a secondary electron (SE) generation mechanism when irradiated by incident lithography electrons. This results in more electrons scattering inside the resist, thus creating a chain reaction of cascading electrons that, upon each collision, exposes the resist in the immediate write area. This has the effect of increasing the overall sensitivity of the resist. It comes at the expense, of a large contribution to the proximity effect.

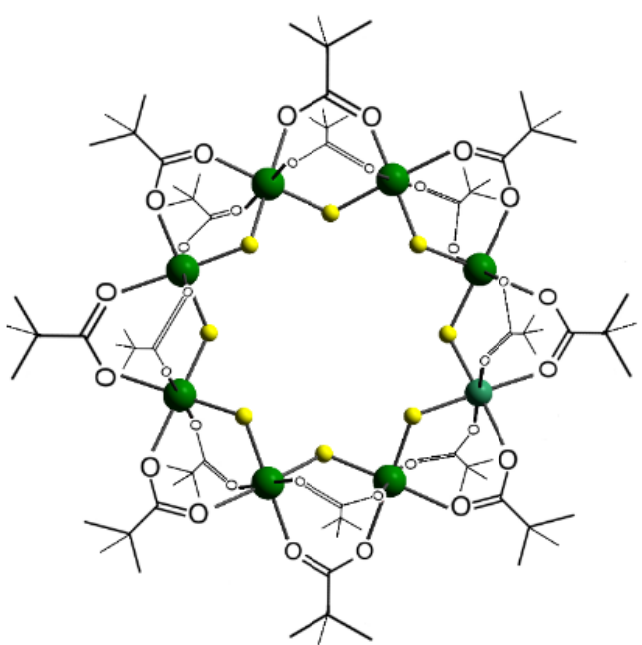

(a)

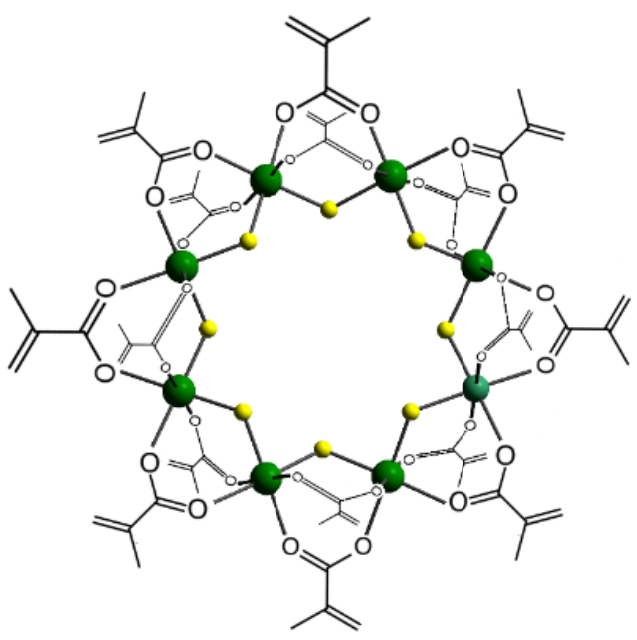

(b)

Figure 1 a) $\mathrm{Cr}_{8} \mathrm{~F}_{8}(\text { Pivalate })_{16}$, b) $\mathrm{Cr}_{8} \mathrm{~F}_{8}$ (Methacrylate $)_{16}$. The structure of the molecules in a crystal, in ball-and-stick representation. Cr atoms are green and $\mathrm{F}$ atoms are yellow. $\mathrm{H}$ atoms are omitted for clarity. 


\section{SIMULATION AND EXPERIMENTAL}

A Monte Carlo simulator was developed at the University of Manchester to gain a physical understanding of the internal electron scattering effects inside the $\mathrm{Cr}_{8} \mathrm{~F}_{8}\left(\mathrm{Pivalate}_{16} \text { and } \mathrm{Cr}_{8} \mathrm{~F}_{8} \text { (methacrylate }\right)_{16}$ resist systems [4, 7]. Unfortunately, no single model accurately describes the electron behaviour in a resist for the energy range of $5 \mathrm{eV}-100$ $\mathrm{KeV}$. The simulator therefore uses two models to describe the electron scattering behaviour. The first utilizes the Joy model, which is based on Rutherford scattering and is an accepted estimate for electrons with kinetic energies above 500 $\mathrm{eV}$, where quantum effects are ignored [8]. With each scattering event, the incident electron loses a portion of its initial energy and very often ends up with an energy below the $500 \mathrm{eV}$ threshold. Therefore, a model for low-energy electrons $(100 \mathrm{eV}$ and below) was also employed. This model uses the hard-sphere potential approximation, which exploits a quantum mechanical approach to electron scattering [9]. It incorporates the use of the partial wave expansion method (PWEM), which is a method for modelling low energy electrons scattering through metals and other solids [10 - 12].

It has been hypothesized that upon a collision event, both the primary electrons (PEs) and SEs experience the statistical chance of exhibiting the correct energy to emit an Auger electron (AE). Understanding the behaviour of these electrons is essential because they will be emitted from inner shells of the scattering atoms and will have a low energy that is suitable for generating even more SEs and AEs (this happens because the AE energy is lower than the first generation of AE emission, resulting in AE generation from the outer shells). Ultimately, this causes a cascading event as AEs travel through the immediate exposure area of the resist. These electrons contribute significantly to the proximity effect and therefore must be evaluated by the simulator. The Auger generation model is based on the work by Ding, which uses the Casnati cross-section for understanding inner shell ionization [13, 14].

The simulation was performed on a $30 \mathrm{~nm}$ layer of each electron beam resist, $\mathrm{Cr}_{8} \mathrm{~F}_{8}(\mathrm{Pivalate})_{16}$ and $\mathrm{Cr}_{8} \mathrm{~F}_{8}(\text { Methacrylate })_{16}$, with an underlying $50 \mathrm{~nm}$ substrate of silicon and chromium, respectively. Table 1 shows the physical properties of each material. The incident electron beam had a Gaussian distribution of $3 \sigma$, where the spot size had a diameter of $2 \mathrm{~nm}$. The simulation was run using 1,000,000 electrons to reduce the statistical error.

\begin{tabular}{|l|c|c|c|c|}
\hline Physical property & $\mathrm{Cr}_{8} \mathrm{~F}_{8}(\text { Pivalate })_{16}$ & $\mathrm{Cr}_{8} \mathrm{~F}_{8}$ (Methacrylate $)_{16}$ & Silicon & Chromium \\
\hline Density $\left(\mathrm{g} / \mathrm{cm}^{3}\right)$ & 1.2 & 1.2 & 2.33 & 7.14 \\
\hline Effective Atomic Number & 9.0 & 9.7 & 14 & 24 \\
\hline Average Atomic Weight $(\mathrm{g} / \mathrm{mol})$ & 2192 & 3794 & 28.0855 & 51.9 \\
\hline Mean Ionization Potential $(\mathrm{eV})$ & 126.4 & 132.8 & 174 & 266.2 \\
\hline
\end{tabular}

Table 1: Physical properties of the resist materials and substrates.

To fabricate a photomask, chromium is typically used as the absorbing photon layer. Therefore, the resist must be deposited on this material and patterned successfully by the EBL tool, followed by transfer of the high-resolution pattern into the $\mathrm{Cr}$ layer. In more detail, a $100 \mathrm{~mm} \mathrm{Si}<100>$ wafer was coated with $\mathrm{Cr}$ using magnetron sputtering in an AJA Orion UHV series sputter system. The wafer process pressure was kept at $3 \mathrm{mtorr}$, as measured by a capacitance manometer utilizing downstream adaptive pressure control. Process gas species flow rates were kept constant at $20 \mathrm{sccm}$ using a mass flow controller. The argon gas used for sputtering was of semiconductor grade purity. The thin film thickness and deposition rate data were measured by a quartz crystal thickness monitor. The system base pressure was 1E-8 torr prior to beginning deposition. The Cr layer was deposited with simultaneous RF substrate etching in order to smooth the RMS roughness. The substrate was pre-sputtered for 60 seconds at $100 \mathrm{~W}$ RF prior to the shutter opening. Deposition parameters included $200 \mathrm{~W}$ DC with a target power density of approximately $10 \mathrm{~W} / \mathrm{cm}^{2}$, to achieve a deposition rate of $1.22 \mathrm{Angstroms} / \mathrm{s}$ and an approximate thickness of $50 \mathrm{~nm}$. The Cr layer was pre-sputtered for 60 seconds prior to shutter opening using the deposition parameters of $230 \mathrm{~W}$ DC with a target power density of approximately $10 \mathrm{~W} / \mathrm{cm}^{2}$, and a deposition rate of 0.58 Angstroms $/ \mathrm{s}$ and an approximate thickness of $100 \mathrm{~nm}$. The 100 W RF substrate etch was continued for the duration of the Chromium deposition and extended to account for backsputtered material caused by the RF power delivered to the substrate. After the magnetron's shutter closed the RF power is ramped down over 40 seconds, resulting in some slight etching without any incoming $\mathrm{Cr}$ flux. The wafer was diced into $20 \mathrm{~mm} \times 20 \mathrm{~mm}$ substrates.

The resist fabrication process is as follows. First, introduce $30 \mathrm{mg}$ of $\mathrm{Cr}_{8} \mathrm{~F}_{8}\left(\right.$ Pivalate $_{16}$ to $3 \mathrm{~g}$ of Hexane and filter it using a $0.2 \mu \mathrm{m}$ PTFE syringe filter. The $\mathrm{Cr}_{8} \mathrm{~F}_{8}$ (Pivalate) ${ }_{16}$ resist was spun onto $20 \mathrm{~mm} \times 20 \mathrm{~mm}$ silicon substrates. The resist was spun using a spin cycle of $6000 \mathrm{rpm}$ for 40 seconds, which was followed by a soft bake at $100^{\circ} \mathrm{C}$ for 2 minutes, allowing the cast solvent to evaporate. The resulting resist film had a thickness of $30 \mathrm{~nm}$. This process was 
repeated for the $\mathrm{Cr}_{8} \mathrm{~F}_{8}$ (Methacrylate $)_{16}$ resist, except the hexane solvent was replaced with anisole. $\mathrm{The} \mathrm{Cr}_{8} \mathrm{~F}_{8}\left(\mathrm{Pivalate}_{16}\right.$ resist was spun onto the $\mathrm{Cr}$-coated silicon substrates using the same process that was used for coating the silicon substrates.

The exposure clearing dose of each resist material was determined from a one-dimensional matrix of a single pixel lines that were $5 \mu \mathrm{m}$ long; the width of the line was the width of the electron beam, i.e. $2 \mathrm{~nm}$. The one-dimensional matrix had each single pixel line separated by a pitch of $60,55,50,45$, and $40 \mathrm{~nm}$. These were exposed with a dose range from 1000 to $80000 \mu \mathrm{C} / \mathrm{cm}^{2}$ in incremental steps of $50 \mu \mathrm{C} / \mathrm{cm}^{2}$. All resists were exposed using a Raith EPBG 5200 electron beam writer. The patterns were exposed using an acceleration voltage of $100 \mathrm{KeV}$, a probe current of $300 \mathrm{pA}$,

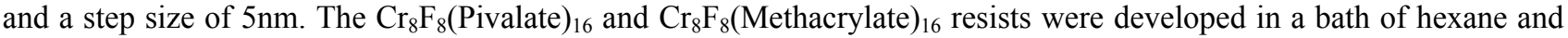
anisole, respectively, for 10 seconds. All samples were blown dry using Nitrogen $\left(\mathrm{N}_{2}\right)$.

\section{RESULTS AND DISCUSSION}

Figure 2 shows a 3D scattering trajectory plot that illustrates that both of the resists confine the primary electrons (PEs) to within a $15 \mathrm{~nm}$ diameter of the immediate write area, suggesting that high resolution nanostructures would be expected. It is evident that $\mathrm{Cr}_{8} \mathrm{~F}_{8}$ (Methacrylate) ${ }_{16}$ resist generates more SEs in the resist (see Figure $2 \mathrm{~b}$ and $\mathrm{d}$ ) than the $\mathrm{Cr}_{8} \mathrm{~F}_{8}\left(\right.$ Pivalate) ${ }_{16}$ resist (see Figure 2a and c).

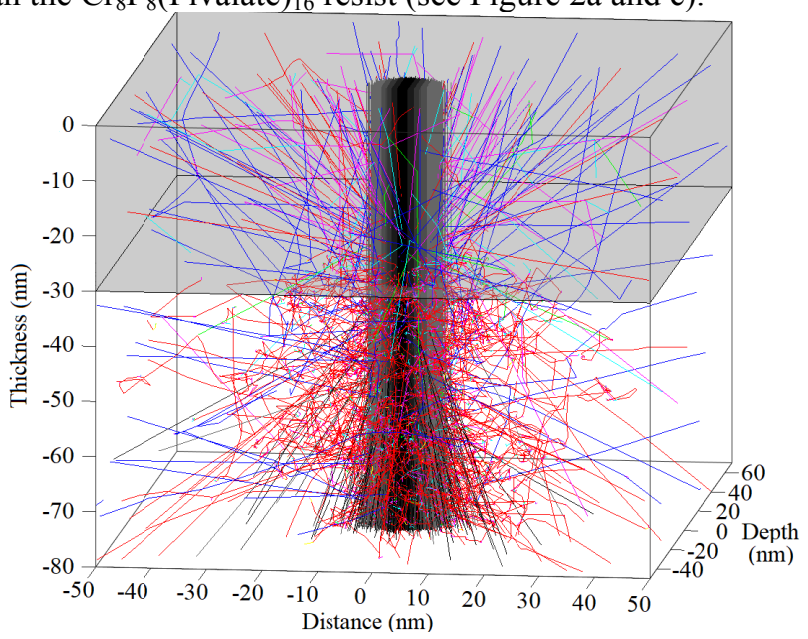

(a)

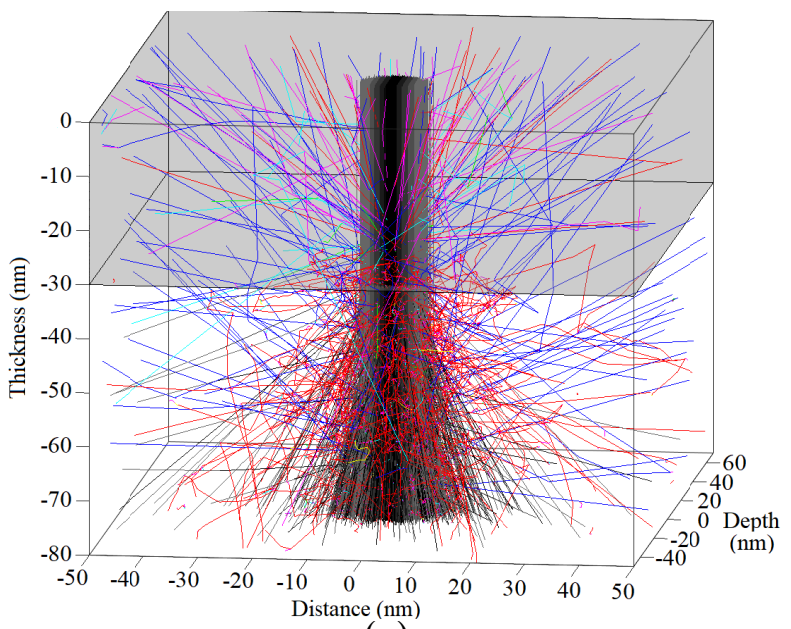

(c)

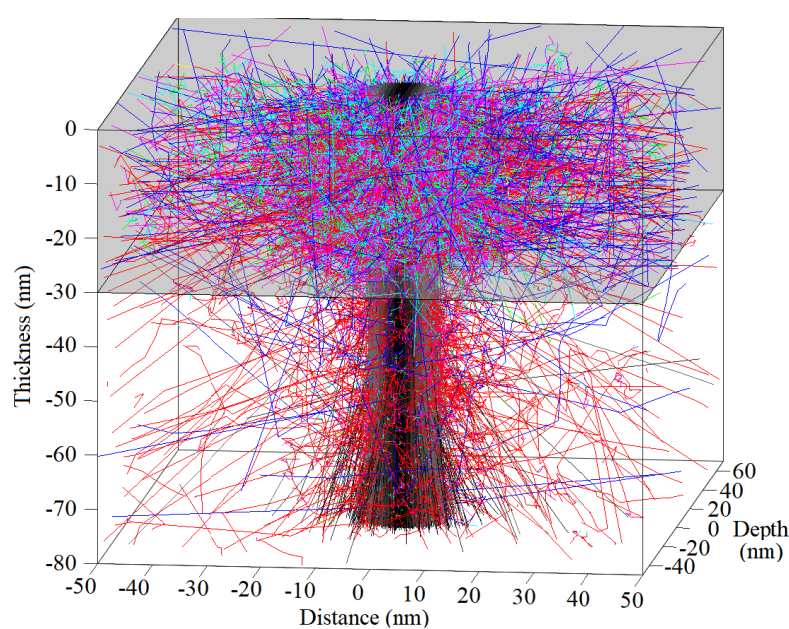

(b)

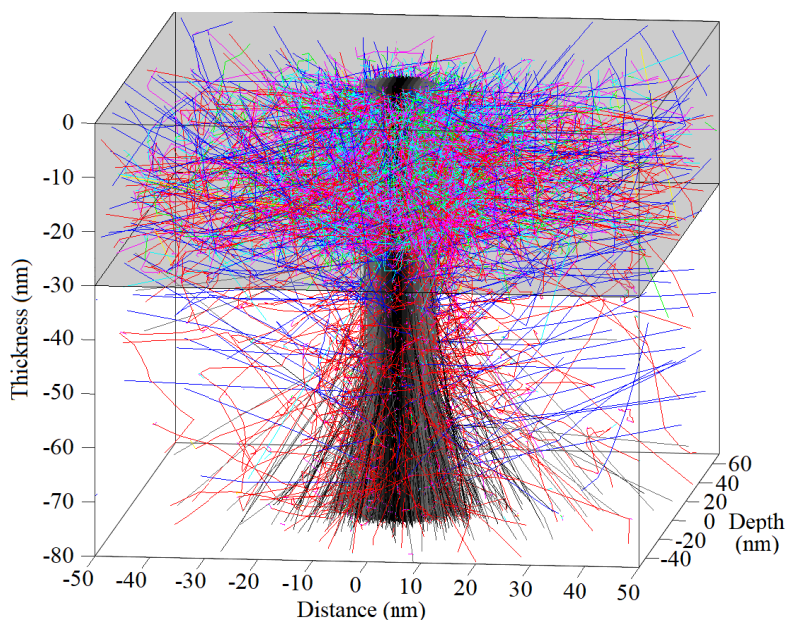

(d)

Figure 2 Point spread function of the internal electron scattering interactions inside (a) $\mathrm{Cr}_{8} \mathrm{~F}_{8}\left(\mathrm{Pivalate}_{16}\right.$ resist on $50 \mathrm{~nm}$ of Si. (b) $\mathrm{Cr}_{8} \mathrm{~F}_{8}$ (Methacrylate $)_{16}$ resist on $50 \mathrm{~nm}$ of $\mathrm{Si}$, (c) $\mathrm{Cr}_{8} \mathrm{~F}_{8}$ (Pivalate) $)_{16}$ resist on $50 \mathrm{~nm}$ of $\mathrm{Cr}$, (d) $\mathrm{Cr}_{8} \mathrm{~F}_{8}(\text { Methacrylate })_{16}$ resist on $50 \mathrm{~nm}$ of $\mathrm{Cr}$. The acceleration voltage used was $100 \mathrm{KeV}$. The black lines represent the PEs from the incident beam while the SEs above 500 $\mathrm{eV}$ are represented by the red lines. The SEs, which have associated energies below $500 \mathrm{eV}$, were generated by first, second and third order collisions and are indicated by purple, cyan and green, respectively. The blue lines are backscattered electrons. One million electrons are inserted into a single spot. 
This is important as these electrons are responsible for exposing the resist and subsequently increase the overall sensitivity while contributing to the proximity effect. When the methacrylate molecule is appended to the outside of the molecule, the dangling alkene groups are induced by the PEs to eject further SEs, which contribute to the reduction of the overall exposure dose. The associated energy of the SEs is considerably lower than that of the PEs, leading to an increased number of scattering events, with subsequent collisions generating even more SEs, thereby producing a cascade of scattering events. This effect can be seen in Figures $2 \mathrm{~b}$ and d, where SEs are scattered at angles larger than $80^{\circ}$ in arbitrary trajectories away from the primary beam, exposing the resist laterally. This is how the SE plays a major role in both widening the size of the nanostructure and producing a more sensitive resist.

Figure 3a shows the number of SEs generated inside each of the resists as a function of acceleration voltage. At the lower energies of 10 to $50 \mathrm{KeV}$, the PE is slow enough to cause multiple inelastic scattering events and generate more SEs, increasing the sensitivity of the resist while sacrificing resolution. At the higher energies of 50 to $100 \mathrm{KeV}$, the PEs have a lower probability of generating SEs and therefore more collisions are required in the resist material to lose sufficient energy and therefore to increase the probability of generating an SE (see Figure 2). As a consequence, a substantial number of incident electrons will come to rest deep in the Si substrate below without having participated significantly in exposing the resist, or otherwise can be backscattered into the underside of the resist material approximately $30-40 \mu \mathrm{m}$ away from the immediate exposure area. Thus, while $100 \mathrm{KeV}$ can achieve high resolution by narrowly confining the forward scattering of electrons inside the resist, this comes at the expense of higher dose and longer writing times. Currently, $50 \mathrm{KeV}$ EBL tools are employed by the semiconductor industry because it best optimizes resolution versus writing time; Figure 3 reflects this philosophy.

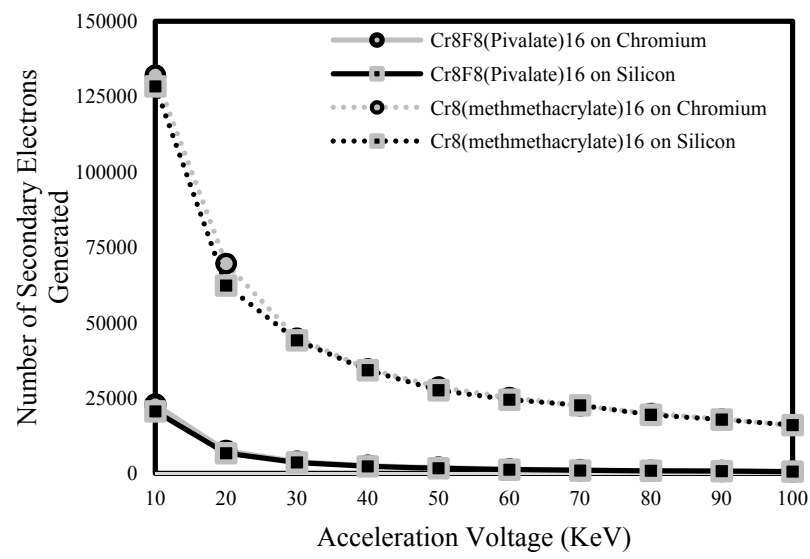

(a)

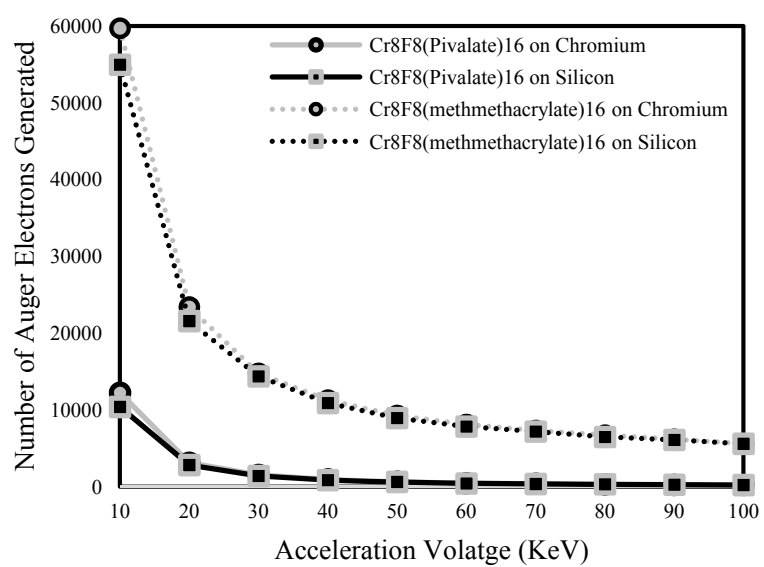

(b)

Figure 3 The number of Electrons generated in $30 \mathrm{~nm}$ thick resist films on $50 \mathrm{~nm}$ thick Si substrate. a) Total number of secondary electrons generated, b) Total number of Auger electrons generated from the resist materials.

It is clear that replacing the pivalate molecule with the methacrylate molecule significantly increases the number of SEs that are generated, thus predicting that the $\mathrm{Cr}_{8} \mathrm{~F}_{8}$ (Methacrylate) ${ }_{16}$ resist will have increased sensitivity compared to $\mathrm{Cr}_{8} \mathrm{~F}_{8}$ (Pivalate) $)_{16}$. This is due to the methacrylate's alkene groups generating SEs in close proximity of $\mathrm{Cr}$ atoms in the resist; $\mathrm{Cr}$ has a larger electron energy stopping power than that of the $\mathrm{C}, \mathrm{O}$ and $\mathrm{H}$ atoms in the organic molecule and therefore reduces the energy of the electron. As the energy reduction of the incident electron passes the threshold of which the probability of a secondary electron being created, they are free to collide with the Alkene groups in the Methacrylate molecule and this will allow more secondary electrons to be generated (their energy will be reduced more than the first generation) and this will create an avalanche effect until their associated energy is lower than $3.6 \mathrm{eV}$ (which is the energy required to scission a $\mathrm{C}-\mathrm{C}$ bond). As a result of the scattering angle the secondary electron penetrates through the $\mathrm{Cr}_{8} \mathrm{~F}_{8}$ (Methacrylate) ${ }_{16}$ resist it exposes it laterally. This has the effect of reducing the resolution of the pitch. 
The number of AEs generated is significant because there is a high probability of emitting low energy AEs from the 1S shell, since the lowest associated energy $(5989 \mathrm{eV})$ for an incident electron shown in Figure $3 \mathrm{~b}$ was $10 \mathrm{KeV}$. The $\mathrm{Cr}$ atom requires the largest associated incident electron energy to emit an $\mathrm{AE}$ in both resist systems because the excitation energy that is essential to ionize the electron from its ground state to a higher orbit is $5989 \mathrm{eV}$. EBL tools that operate at higher incident electron energies of the $\mathrm{Cr}$ electronic ground state are therefore able to take advantage of this process. It must be noted that EBL tools that use large acceleration voltages (50 KeV and above) will have a lower probability of emitting an $\mathrm{AE}$ because the scattering interaction is lower and this can be seen in Figure $3 \mathrm{~b}$. It is evident that incident electrons with low energies emit more AEs because the emitted AEs have low energy with respect to the energy of the incident electron, thereby increasing the statistical chance of creating further secondary and secondary Auger electrons (if the excitation energy of the secondary AEs is correct) upon each collision. Hence causing a chain reaction of cascading electrons in the immediate exposure area, leading to a significant reduction in exposure dose. It is clear that the dangling alkene groups of the methacrylate molecule are induced by all types of electrons (PEs, SEs and AEs) and generate significantly more AEs than that of the $\mathrm{Cr}_{8} \mathrm{~F}_{8}$ (Pivalate $)_{16}$ resist because they have an electron from the carbon atom that is free to be ionized from the ground state (this requires an excitation energy of $284.2 \mathrm{eV}$ and the probability of generated SEs and emitted AEs having this discrete energy is high).

To build on the result and the explanation of Figure 3, Figure 4 shows the number of SEs and AEs that are generated in the $\mathrm{Cr}_{8} \mathrm{~F}_{8}$ (Methacrylate) ${ }_{16}$ resist compared to the number generated in the $\mathrm{Cr}_{8} \mathrm{~F}_{8}$ (Pivalate $)_{16}$ resist, expressed as a ratio. This ratio was calculated to communicate the overall improvement in exposure performance of the alkene groups compared to the pivalates, which are expected to be less sensitive. It was found that the presence of the methacrylate molecule increased the sensitivity of the resist material, as predicted, thereby reducing the exposure doses required to produce a pattern. This is another way of expressing the results previously given in Figure 2 and 3, which show the same overall effect.

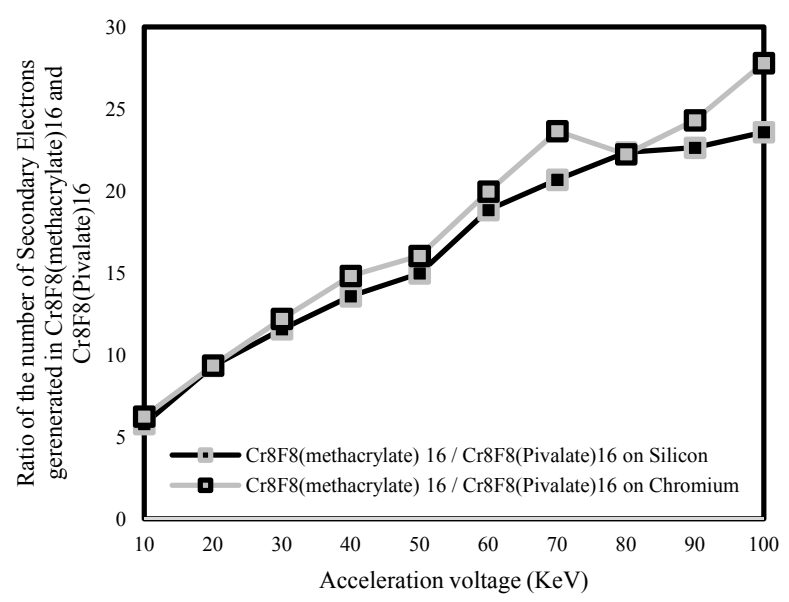

(a)

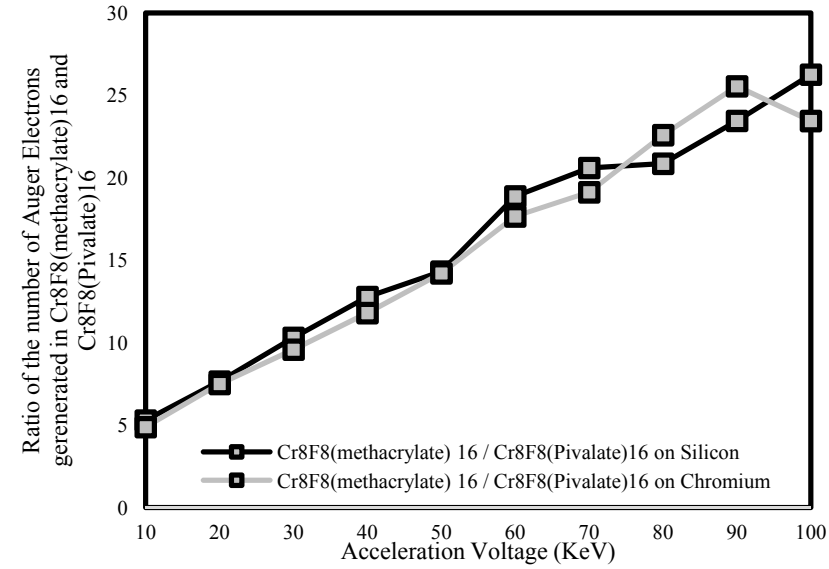

(b)

Figure 4. a) The ratio of secondary electrons generated by $\mathrm{Cr}_{8} \mathrm{~F}_{8}(\text { Methacrylate })_{16}$ and $\mathrm{Cr}_{8} \mathrm{~F}_{8}\left(\mathrm{Pivalate}_{16}\right.$ resists on $50 \mathrm{~nm} \mathrm{Si}$ and $\mathrm{Cr}$ with varying acceleration voltage, b) The ratio of Auger electrons generated by $\mathrm{Cr}_{8} \mathrm{~F}_{8}(\text { Methacrylate })_{16}$ and $\mathrm{Cr}_{8} \mathrm{~F}_{8}\left(\mathrm{Pivalate}_{16}\right.$ resists on Siand $\mathrm{Cr}$ with varying acceleration voltage.

Compared to pivalate, methacrylate increases the number of generated SEs and AEs by a factor of 3.7 and 4.9 , respectively, over the acceleration voltage range presented. The amplifications seen here increase the speed of the write times by increasing the solubility of the molecule upon the development process. Thus, to achieve the maximum resolution while avoiding overexposure, the exposure dose must be decreased to compensate for the extra concentration of electrons in the immediate exposure area. From this, the maximum write speed that can be theoretically achieved by $\mathrm{Cr}_{8} \mathrm{~F}_{8}(\text { Methacrylate })_{16}$ is approximately 23.6 times faster than $\mathrm{Cr}_{8} \mathrm{~F}_{8}(\text { Pivalate })_{16}$ at $100 \mathrm{KeV}$. This particular acceleration voltage is of interest as it was used to validate theoretical results with experimental results, which are presented next.

Figure 5 shows scanning electron microscope (SEM) micrographs of developed nanostructures written into $\mathrm{Cr}_{8} \mathrm{~F}_{8}(\text { Pivalate })_{16}$ and $\mathrm{Cr}_{8} \mathrm{~F}_{8}(\text { Methacrylate })_{16}$ resists. It is evident that $\mathrm{Cr}_{8} \mathrm{~F}_{8}$ (Methacrylate $)_{16}$ required an exposure dose lower than $\mathrm{Cr}_{8} \mathrm{~F}_{8}$ (Pivalate) $)_{16}$ due to the introduction of the alkene groups. Even though the clearing dose of $\mathrm{Cr}_{8} \mathrm{~F}_{8}(\text { Methacrylate })_{16}$ is considerably reduced, Figure $5 \mathrm{~b}$ clearly shows that the nanostructures could only be resolved at 
a pitch $60 \mathrm{~nm}$ (and no lower) as predicted by the results of the simulations (see Figure 3), while $\mathrm{Cr}_{8} \mathrm{~F}_{8}\left(\mathrm{Pivalate}_{16}\right.$ produced a pattern with a pitch as small as $40 \mathrm{~nm}$. Figure $5 \mathrm{c}$ shows $15 \mathrm{~nm}$ features written into $\mathrm{Cr}_{8} \mathrm{~F}_{8}\left(\mathrm{Pivalate}_{16}\right.$ resist on $100 \mathrm{~nm}$ of Cr. Interestingly, only a $45 \mathrm{~nm}$ pitch could be achieved on those samples; this is because $\mathrm{Cr}$, compared to $\mathrm{Si}$ only, leads to a higher number of BSEs emitted from the substrate layer back into the underside of the resist, contributing to the proximity effect. This is expected because the atomic number of $\mathrm{Cr}$ is 1.71 larger than that of $\mathrm{Si}$; this is predicted by the results of the simulation.

(a) $\mathrm{Cr}_{8} \mathrm{~F}_{8}$ (Pivalate) ${ }_{16}$ on $\mathrm{Si}$

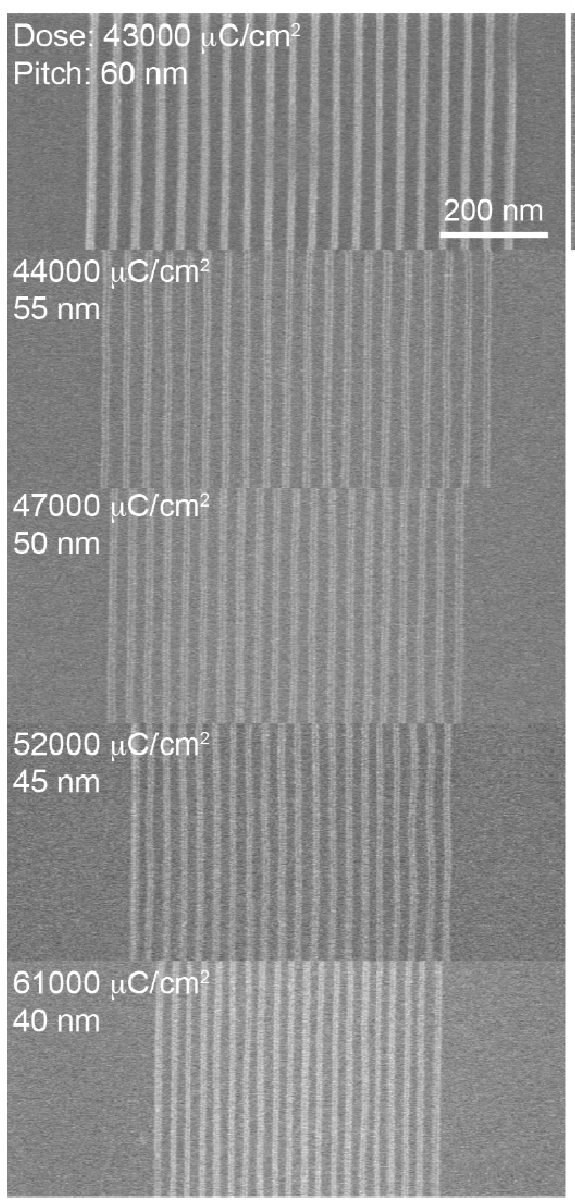

(b) $\mathrm{Cr}_{8} \mathrm{~F}_{8}(\text { Methacrylate })_{16}$ on $\mathrm{Si}$

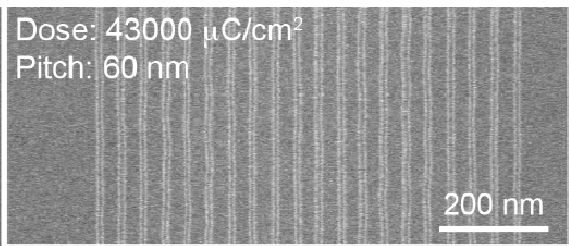

(c) $\mathrm{Cr}_{8} \mathrm{~F}_{8}$ (Pivalate) $)_{16}$ on $\mathrm{Cr} / \mathrm{Si}$

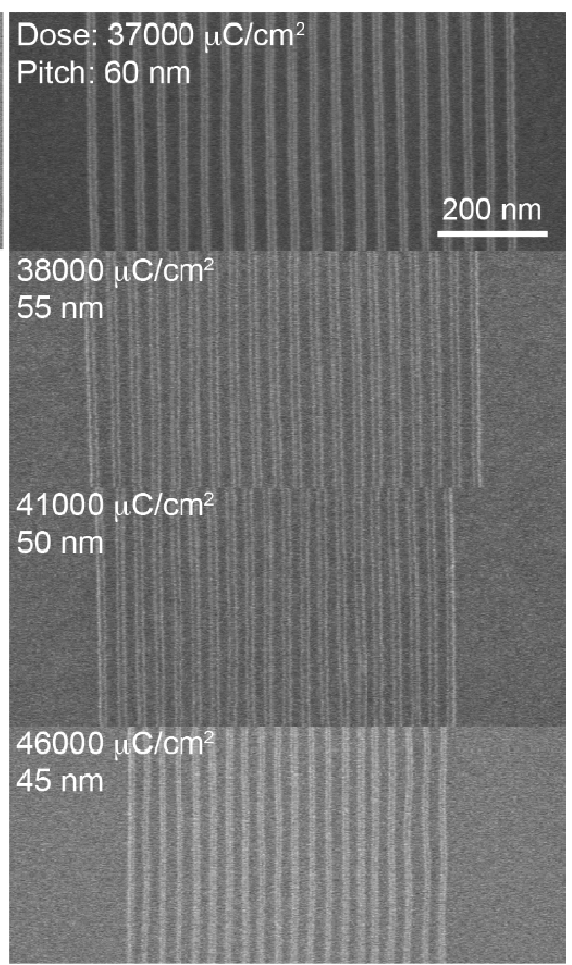

Figure 5 Effect of electron doses versus pitch on $\mathrm{Si}$ and $\mathrm{Cr}$ substrate materials. (a) Top down view of $15 \mathrm{~nm}$ lines on a $60,55,50,45$ and $40 \mathrm{~nm}$ pitch fabricated in $\mathrm{Cr}_{8} \mathrm{~F}_{8}$ (Pivalate) ${ }_{16}$ resist on Si substrate. (b) Top down view of $15 \mathrm{~nm}$ lines on a $60 \mathrm{~nm}$ pitch fabricated in $\mathrm{Cr}_{8} \mathrm{~F}_{8}$ (methacrylate) ${ }_{16}$ resist on $\mathrm{Si}$ substrate. (c) Top down view of $15 \mathrm{~nm}$ lines on a $60,55,50 \mathrm{~nm}$ pitch fabricated in $\mathrm{Cr}_{8} \mathrm{~F}_{8}\left(\mathrm{Pivalate}_{16}\right.$ resist on $100 \mathrm{~nm}$ of $\mathrm{Cr}$ on $\mathrm{Si}$ substrate.

Figure 6 shows how the exposure clearing doses vary with pitch of the pattern and confirms the role of the methacrylate molecules acting as $\mathrm{SE}$ and $\mathrm{AE}$ generators. The clearing dose in $\mathrm{Cr}_{8} \mathrm{~F}_{8}$ (Methacrylate) ${ }_{16}$ is 22.6 lower than that in $\mathrm{Cr}_{8} \mathrm{~F}_{8}(\text { Pivalate })_{16}$, showing strong agreement with the factor of 23.6 predicted by simulation. The $4.3 \%$ simulation error is likely due to the Si substrate only having a thickness of $50 \mathrm{~nm}$, whereas in the experiment, the substrate is 550 $\mu \mathrm{m}$ thick. The pattern exposure took place using incident electrons that had an acceleration voltage was $100 \mathrm{KeV}$; at this energy, the electrons can penetrate as deeply as $40 \mu \mathrm{m}$ into the Si substrate and generate BSEs that get deposited into the underside of the resist, thus lowering both the exposure dose required and the patterning resolution.

It must be pointed out here again that the Monte Carlo simulator uses two models; the semi classical model accounts for electrons with energies of $500 \mathrm{eV}$ and above and the hard sphere method models quantum effects for electron energies of $100 \mathrm{eV}$ and below. Clearly, some electrons may have energy in the $400 \mathrm{eV}$ gap between the models, and are therefore unaccounted for. The simulator also does not account for x-rays generated by the electrons. These xrays can cause bond scissions in the immediately exposure area and have the effect of lowering the exposure dose. 


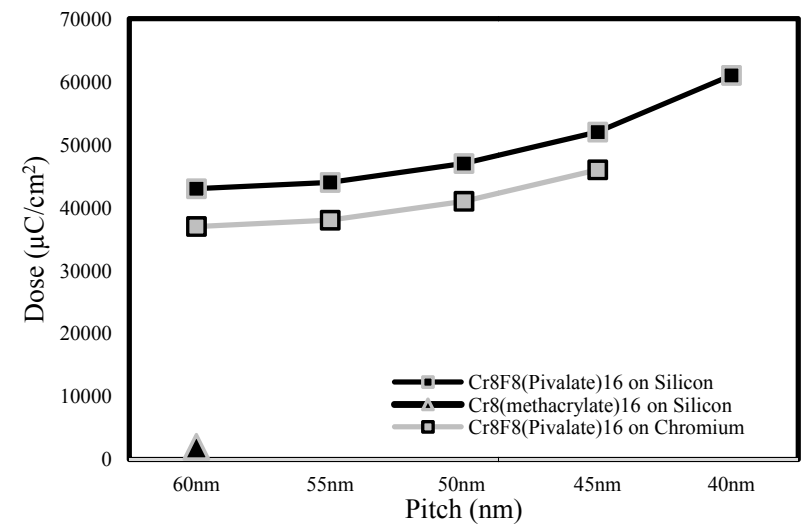

Figure 6 Electron exposure doses for the two resist materials on Si and $\mathrm{Cr}$ substrates.

Figure 7a shows $15 \mathrm{~nm}$ nanostructures with a $40 \mathrm{~nm}$ pitch that have been patterned in the $\mathrm{Cr}_{8} \mathrm{~F}_{8}(\mathrm{Pivalate})_{16}$ resist on a Si substrate. The resist exhibited extremely high dry etch selectivity when compared with Si. Since it is difficult to distinguish between the resist and $\mathrm{Si}$ in the micrographs, the Si was etched with conditions that created an undercutting effect, where the resist-Si interface can clearly be seen. Although this helps with characterization, it must be noted that this kind of undercut is an undesirable result in real FinFET fabrication.

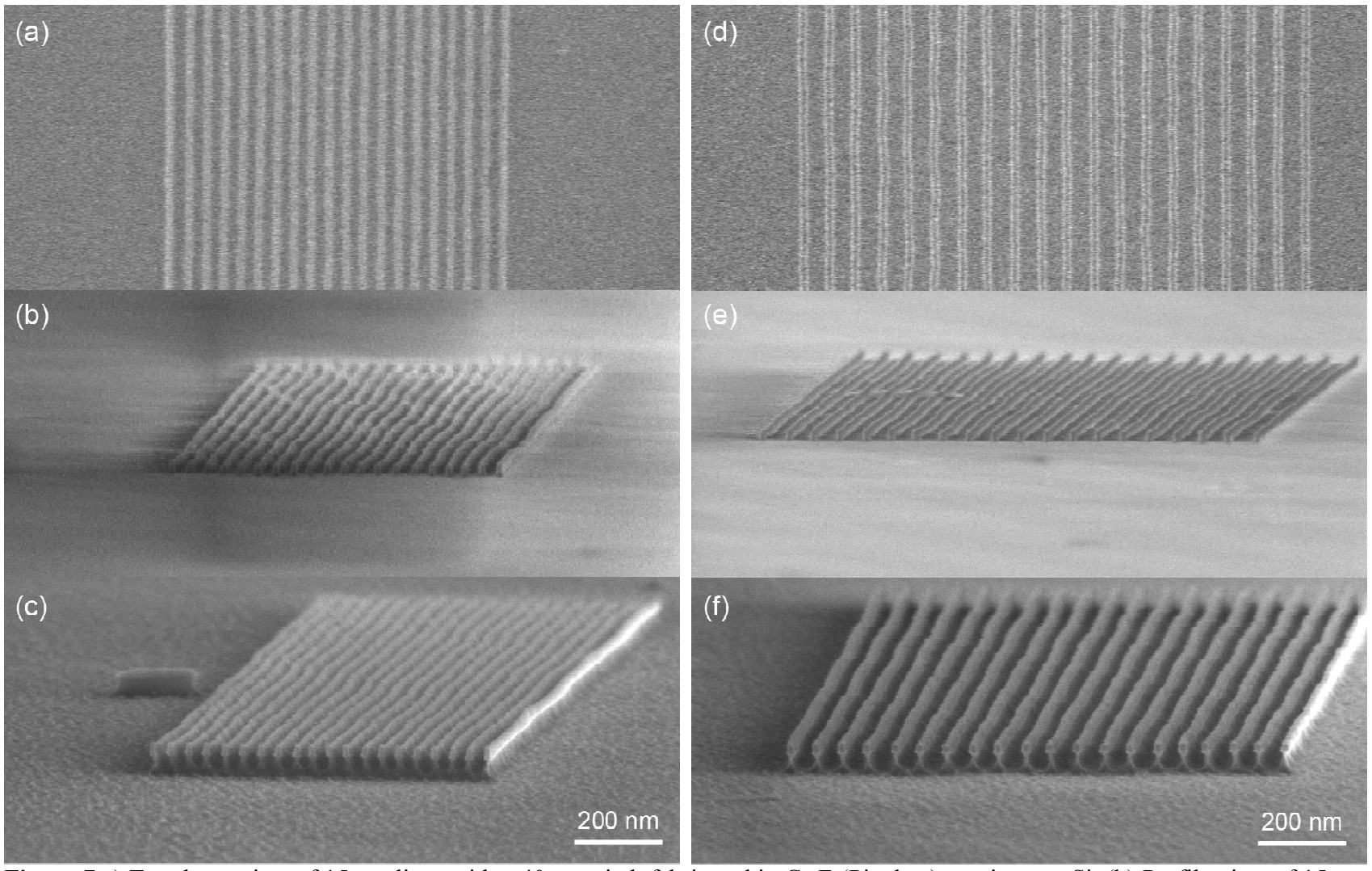

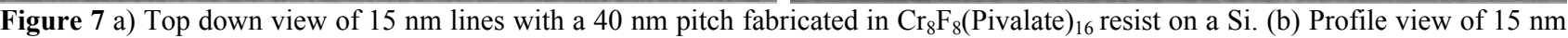

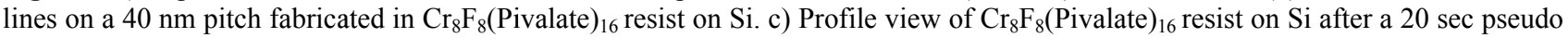
Bosch dry etch process. d) Top down view of $15 \mathrm{~nm}$ lines on a $60 \mathrm{~nm}$ pitch fabricated in $\mathrm{Cr}_{8} \mathrm{~F}_{8}\left(\mathrm{Methacrylate}_{16}\right.$ resist on Si. (e) Profile view of $15 \mathrm{~nm}$ lines on a $60 \mathrm{~nm}$ pitch fabricated in $\mathrm{Cr}_{8} \mathrm{~F}_{8}$ (Methacrylate $)_{16}$ resist on Si. f) Profile view of $\mathrm{Cr}_{8} \mathrm{~F}_{8}\left(\mathrm{Methacrylate}_{16}\right.$ resist on Si substrate after a $20 \mathrm{sec}$ pseudo Bosch dry etch process. Parameters: $\mathrm{SF}_{6}$ and $\mathrm{C}_{4} \mathrm{~F}_{8}$ gases were used with flow rates of 22 and 35 sccm, respectively; the deep reactive ion etching (DRIE) forward power was $20 \mathrm{~W}$ and the ICP forward power was $1200 \mathrm{~W}$. 
The underlying Silicon was etched with a pseudo Bosch dry etch process that uses ICP of $\mathrm{SF}_{6}$ and $\mathrm{C}_{4} \mathrm{~F}_{8}$ gases. Before the etching process, Figure $7 \mathrm{~b}$ shows that the resist produced a resolution of $15 \mathrm{~nm}$ while exhibiting a height of $25.4 \mathrm{~nm}$. Figure 7c shows the resolution of the Si nanostructures after the etch was $11 \mathrm{~nm}$, while exhibiting a height of $36.3 \mathrm{~nm}$. The remaining resist thickness is measured to be $24.6 \mathrm{~nm}$, which leads to an effective resist etch rate of 0.04 $\mathrm{nm} / \mathrm{sec}$ over the 20 seconds of etch time, while the $\mathrm{Si}$ etch rate was $1.8 \mathrm{~nm} / \mathrm{sec}$. These measurements indicate a selectivity of 45:1.That is, the silicon etches 45 times faster than the resist. Figure $7 \mathrm{~d}$ shows $15 \mathrm{~nm}$ nanostructures with a $60 \mathrm{~nm}$ pitch that have been patterned in the $\mathrm{Cr}_{8} \mathrm{~F}_{8}$ (Methacrylate) ${ }_{16}$ resist on Si substrate; this was the best resolution that was obtained. Figure $7 \mathrm{e}$ shows $15 \mathrm{~nm}$ nanostructures with a $60 \mathrm{~nm}$ pitch while exhibiting a height of $22.5 \mathrm{~nm}$. Figure $7 \mathrm{f}$ shows the width of Si nanostructures after the etch to be $16 \mathrm{~nm}$, while exhibiting a height of $43 \mathrm{~nm}$. The remaining resist thickness is measured to be $21.8 \mathrm{~nm}$, which leads to the resist etch rate of $0.035 \mathrm{~nm} / \mathrm{sec}$, while the Si etch rate was 2.15 $\mathrm{nm} / \mathrm{sec}$. This indicates a selectivity of $61.4: 1$. This is approximately equivalent to the selectivity that is achieved with aluminum oxide masks [15], which were to this point the best masks available (it should be noted that the use of aluminum oxide masks requires more processing steps and has increased costs compared to this process). It is evident that the additives of the methyl methacrylate do not affect the etching performance of this material; clearly the chromium oxide does not react with the $\mathrm{SF}_{6}$ or $\mathrm{C}_{4} \mathrm{~F}_{8}$ gases. As the gases come into contact with the resist, there is no chemical reactivity, thus the resist keeps its structural integrity and yields a large selectivity. This is significant because other resists such as PMMA, ZEP520A and HSQ have etch selectivities of only 2:1, 2.89:1 and 4.16:1, respectively [16]. That is, the highest dry etch resistance among these commonly used resists is only a little over 4 times that of $\mathrm{Si}$. The new material reported here outperforms each of these materials by a factor of at least $\sim 14.75$.

At first glance, it appears that the Si etching process for the $\mathrm{Cr}_{8} \mathrm{~F}_{8}(\text { Pivalate })_{16}$ resist exhibits a poorer etch selectivity when compared to the $\mathrm{Cr}_{8} \mathrm{~F}_{8}$ (Methacrylate) ${ }_{16}$ resist. This is not the case, because the pitch was $20 \mathrm{~nm}$ smaller and therefore the etch efficiency was decreased, which inherently decreased the etch rate of the underlying substrate and produced a smaller etch depth, leading to a decrease in the selectivity. An experiment in which the pitch is extended to 100 or $200 \mathrm{~nm}$ is expected to show equal or near-equal etch selectivity, once the small pitch effect is mitigated [4].

Figure 8a shows $15 \mathrm{~nm}$ lines on a $50 \mathrm{~nm}$ pitch that have been patterned in the $\mathrm{Cr}_{8} \mathrm{~F}_{8}$ (Pivalate) ${ }_{16}$ resist on a 100 $\mathrm{nm} \mathrm{Cr}$ layer that was deposited on Si substrate. The underlying Cr layer was etched with a dry etch process that uses an ICP of $\mathrm{Cl}_{2}$ and $\mathrm{O}_{2}$ gases. Before the etching process, Figure $8 \mathrm{~b}$ shows that the resist yielded line widths of $15 \mathrm{~nm}$ while exhibiting a height of $26.7 \mathrm{~nm}$. Figure 8c shows the width of the Cr nanostructures after the etch was $15 \mathrm{~nm}$, while exhibiting a height of $20 \mathrm{~nm}$. The profile of these Cr nanostructures are triangular in shape, likely due to over etching. The resist has been totally eroded away, which leads to a calculated resist etch rate of $1.33 \mathrm{~nm} / \mathrm{sec}$, while the $\mathrm{Cr}$ etch rate was $1.0 \mathrm{~nm} / \mathrm{sec}$. This yields a selectivity of $0.75: 1$, meaning that $\mathrm{Cr}$ etches 0.25 times slower than $\mathrm{Cr}_{8} \mathrm{~F}_{8}\left(\mathrm{Pivalate}_{16}\right.$ resist with this etch. This is not a surprising result because after electron beam exposure, the $\mathrm{Cr}_{8} \mathrm{~F}_{8}\left(\mathrm{Pivalate}_{16}\right.$ resist is converted into a chromium oxide material and the chemical reaction rate of the $\mathrm{Cl}_{2}$ gas with the chromium oxide is expected to be high.

\section{CONCLUSION}

In summary, two metal organic negative tone electron beam resist have been investigated. It was shown by the Monte Carlo simulations that the $\mathrm{Cr}_{8} \mathrm{~F}_{8}$ (Methacrylate $)_{16}$ resist material generated a significant number of secondary and Auger electrons within the resist when compared to the $\mathrm{Cr}_{8} \mathrm{~F}_{8}\left(\right.$ Pivalate $_{16}$ resist, due to the presence of alkene groups. It was found that $\mathrm{Cr}_{8} \mathrm{~F}_{8}$ (Pivalate) ${ }_{16}$ resist produced very high-resolution nanostructures (15 nm in width) while exhibiting a pitch of $40 \mathrm{~nm}$. This high resolution came at the expense of sensitivity, however; the clearing dose of this resist material was $61000 \mu \mathrm{C} / \mathrm{cm}^{2}$ at $40 \mathrm{~nm}$ pitch and $43000 \mu \mathrm{C} / \mathrm{cm}^{2}$ at $60 \mathrm{~nm}$ pitch. It was shown that the low sensitivity was dramatically improved by replacing the pivalate component with a methacrylate molecule; the clearing dose of this altered resist material was $1900 \mu \mathrm{C} / \mathrm{cm}^{2}$ at $60 \mathrm{~nm}$ pitch ( $40 \mathrm{~nm}$ pitch was not achievable with this material). Substituting methacrylate for pivalate, therefore, resulted in a 22.6 fold increase in resist sensitivity. This experimental result was in strong agreement with simulations, which predicted an increase by a factor of 23.6. Preliminary studies showed that both resists have a remarkable pseudo Bosch dry etch resistance compared to silicon (selectivity was calculated to be 61:1 for the methacrylate resist and 45:1 for the pivalate resist). The high etch resistance is due to the presence of the d-block metal $(\mathrm{Cr})$ in the initial resist, which ultimately yields a chromium oxide hard mask following exposure to an electron beam and subsequent development in solvent. 


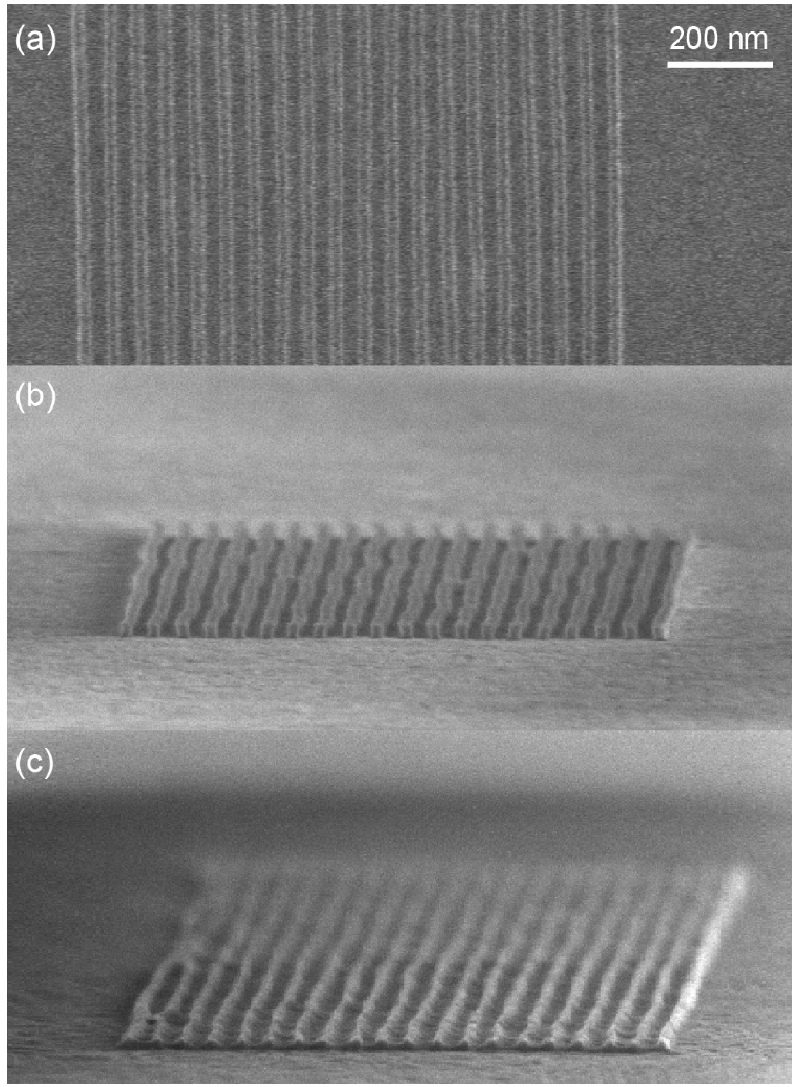

Figure 8 a) Top down view of $15 \mathrm{~nm}$ lines with a $50 \mathrm{~nm}$ pitch fabricated in $\mathrm{Cr}_{8} \mathrm{~F}_{8}$ (Pivalate) $)_{16}$ resist on a $100 \mathrm{~nm} \mathrm{layer} \mathrm{of} \mathrm{Cr} \mathrm{on} \mathrm{Si}$ substrate. (b) Profile view of the nanostructures shown in (a). (c) Profile view of the nanostructures following a 20 sec pseudo Bosch dry etch process, showing fins with a $8 \mathrm{~nm}$ width. Parameters: $\mathrm{Cl}_{2}$ and $\mathrm{O}_{2}$ gases were used with flow rates of 100 and 3 sccm, respectively; the DRIE forward power was $100 \mathrm{~W}$ and the ICP forward power was $1000 \mathrm{~W}$.

\section{ACKNOWLEDGEMENTS}

We acknowledge the EPSRC(UK) for funding (grant EP/R023158/1). The University of Manchester also supported this work. The authors gratefully acknowledge critical support and infrastructure provided for this work by the Kavli Nanoscience Institute at Caltech.

\section{REFERENCES}

[1] van Dodewaard, A. J., Roes, R. F. M., Kwinten, J. A. J., van Delft, F. C. M J. M., van Run, A. J., van LangenSuurling, A. K., Romijn, J., 'Comparison of Negative tone resists NEB22 and UVN30 in E-beam lithography', Microelectronic engineering $53(1-4) 461-464$ (2000).

[2] Masnyj, Z., Mangat, P., Ainley, E., Nordquist, K., Resnick, D., 'Evalution of negative DUV UVN30 for electron beam exposure of NGL masks', Proc. SPIE 3997, Emerging technologies IV, 0277-786x, (2000).

[3] Klein, C., Loeschner, H., Platzgummer, E., ' $50 \mathrm{KeV}$ electron multibeam mask writer for the $11 \mathrm{~nm}$ HP node: first results of the proof of concept tool (eMET POC)', Proc SPIE 8323, Alternative Lithographic Technologies IV, $83230 \mathrm{G}(2012)$.

[4] Lewis, S. M., Fernandez, A., DeRose, G. A., Hunt, M. S., Whitehead, G. F. S., Lagzda, A., Alty, H. R., FerrandoSoria, J., Varey, S., Kostopoulos, A. K., Schedin, F., Muryn, C. A., Timco, G. A., Scherer, A., Yeates, S. G., and Winpenny, R. E. P., "Use of Supramolecular Assemblies as Lithographic Resists", Angew. Chem. Int. Ed., 56, (24), 6749-6752 (2017). 
[5] Noto, V. D., Boeer, A. B., Lavina, S., Muryn, C. A., Bauer, M., Timco, G. A., Negro, E., Rancan, M., Winpenny, R. E. P., and Gross, S., "Functional chromium wheel-based hybrid organic-inorganic materials for dielectric applications', Adv. Funct. Mater. 2009, 19, 3226-3236, (2009).

[6] Laye, R. H., Larsen, F. K., Overgaard, J, Muryn, C. A, McInnes, E. J. L., Rentschler, E., Sanchez, V., Teat, S. J., Güdel, H. U., Waldmann, O., Timco, G. A., and Winpenny, R. E. P., "A family of heterometallic wheels containing potentially fourteen hundred siblings", Chem. Commun., , 1125-1127 (2005).

[7] Lewis, S. M, and DeRose, G. A., Frontiers of Nanoscience, Materials and Processes for Next Generation Lithography, Elsevier, 421, 12, (2016).

[8] Joy, D. C. Monte Carlo Modeling for Electron Microscopy and Microanalysis. New York: Oxford University Press, 1995.

[9] Sakurai, J. J, and Tuan, San Fu. Modern Quantum Mechanics. Menlo Park, California; Wokingham: BenjaminCummings, 1985.

[10] Wolff, P.A., "Theory of Secondary Electron Cascade in Metals", Physical Review, 95 (1) (1954)

[11] Shimizu, R., and Ze-Jun, D., "Monte Carlo modelling of electron-solid interactions", Rep. Prog. Phys. 487531(1992).

[12] Zianni, X., Velessiotis, D., Glezos, N., Trohidou, K. N., "Application of the partial wave expansion method in 3-D low energy electron beam lithography simulation”, Microelectronic Engineering, 57-58: 297-302 (2001).

[13] You, D. S., Li, H. M., and Ding, Z. J., "Monte Carlo simulation of Auger electron emission from thin film on substrate" J. Electron Spectrosc. Relat. Phenom., , 222, 156-161 (2018).

[14] Casnati, E., Tartari, A., and Baraldi, C., "An empirical approach to K-shell ionisation cross section by electrons", J. Phys. B: At. Mol. Phys. 15155 (1982).

[15] Henry, D., S. Walavalkar, S., Homyk, A., Scherer, A., "Alumina etch masks for fabrication of of high-aspect-ratio silicon micropillars and nanopillars", Nanotechnology, 20, (25), 255305 (2009).

[16] A. Goodyear, M. Boettcher, I. Stolberg, M. Cooke, 'Direct comparisoin of the performance of commonly used ebeam resists during nanoscale plasma etching of $\mathrm{Si}, \mathrm{SiO}_{2}$ and $\mathrm{Cr}$ ', Proc. SPIE 9428, 94280V, (2015). 